\title{
A NON-ECLIPSING BINARY MODEL OF THE SYMBIOTIC STAR AG DRA
}

\author{
T. Iijima \\ Asiago Astrophysical Observatory \\ I-36012 Asiago (Vicenza) ITALY
}

\begin{abstract}
The light variation of AG Dra is considered as a result of a variation of free-free emission from a gaseous envelope around the hot component. The emission measure of the envelope at a light maximum is estimated to be about $\mathrm{Ne}^{2} \mathrm{~V}=6.5 \times 10^{59} \mathrm{~cm}^{-3}$. The amplitude of the light variation in the $U$ band should be less than $1.4 \mathrm{mag}$. which is consistent with the observed results. The emission measure of the envelope might vary according to a variation of mass transfer rate in an elliptical binary system.
\end{abstract}

\section{Introduction}

AG Dra (BD $\left.+67^{\circ} 922\right)$ is sometimes called as a yellow symbiotic star, because it has a relatively early type (K $3 \sim 5 \mathrm{III}$ ) cool component (Doroshenko and Nikolov, 1967; Boyarchuk, 1969; Belyakina, 1969; Viotti et al., 1983; etc.). Many works made in the recent years covering the regions from $X$ ray to infrared seem to support a binary model consisting of a $\mathrm{K}$ type giant and a hot compact star (Belyakina, 1969; Anderson et al., 1982; Taranova and Yudin, 1982; Kenyon and Webbink, 1984; Viotti et al., 1983; 1984). Meinunger (1979) found out that the luminosity in the $\mathrm{U}$ band periodically varies with the following elements,

$$
\mathrm{JD}(\operatorname{Max})=2438900+554 \text { (days) } \times \mathrm{E} .
$$

The light curve is different from those of usual eclipsing binaries, namely the light minima are wide and there are significant fluctuations in their depth and phase (Meinunger, 1979; Oliversen and Anderson, 1982). After the large outburst in $1980-81$ an anticipated light minimum on JD 2445326 and a maximum on JD 2445549 were not detected (Luthardt, 1985), which means that the light variation was strongly disturbed by the outburst. On the other hand, the light variation according to the elements of Meinunger (1979) reappeared in the new quiescent stage in 1983-84, that is a light minimum was observed on JD 2445850 (Phase 0.55) and it was again wide (Luthardt, 1985). In this paper a possible model to explain these phenomena is discussed. 


\section{Mode1s}

Viotti et al. (1984) proposed an eclipsing binary model and suggested that the wide light minima may be due to a reflection effect of the radiation from the hot component by the cool component. It seems difficult, however, to explain the complete disappearance of the periodical light variation with such models, because eclipsing phenomena are usually detectable also during outburst stages (e.g. those of CI Cyg: Belyakina, 1983). Taranova and Yudin (1982) proposed another model in which the 1 ight variation is due to a variation of free-free $(f-f)$ emission from a pulsating gaseous envelope around the hot component. Since the envelope may be disturbed by outbursts, the light variation soon after the outburst is in agreement with this model. The reappearance of the same light variation, however, seems to be inconsistent with this model, because after the large disturbance very probably a new pulsation will start with a different mode. It might be possible to overcome these difficulties with an elliptical binary model, in which the 1 ight variation is due to a variation of $f-f$ emission from a gaseous envelope. The envelope, however, does not pulsate by itself, but varies according to a variation of mass transfer in the elliptical binary system. The envelope can be strongly disturbed by outbursts, but since the physical parameters of the binary are not changed, the same light variation will start again in the new quiescent stage.

Viotti et al. (1984) showed that the continuum radiation of AG Dra in the IUE ultraviolet region consists of two components. The one, which is dominant in the region $\lambda<2000 \mathrm{~A}$, has a steep energy distribution corresponding to a black-body curve whereas the other one shows a rather flat energy distribution. They suggested that these components may correspond to the hot compact star and the accretion disk, respectively. It is also possible, however, to construct a model of AG Dra assuming that the latter component is due to $f-f$ emission from a gaseous envelope, as follows. The intensity of the flat component is $1.3 \times 10^{31} \mathrm{erg} \mathrm{sec}^{-1} \mathrm{~A}^{-1}$ at $2860 \mathrm{~A}$ (Viotti et al., 1984), where the distance to AG Dra is 700 pc (Anderson et a1., 1981). The contribution from the hot compact star $\left(R=0.02 R_{\odot}\right.$, $\mathrm{T}=150000 \mathrm{~K}$ : Iijima, 1987) to the continuum is about $10 \%$ of the observed flux and that from the cool component ( $\mathrm{K} 5 \mathrm{III}$ ) is nearly the same. Assuming that $1.1 \times 10^{31} \mathrm{erg} \mathrm{sec}^{-1} \mathrm{~A}^{-1}$ is due to the $f-f$ emission, an emission measure $\mathrm{N}_{\mathrm{e}}^{2} \mathrm{~V}=6.5 \times 10^{59} \mathrm{~cm}^{-3}$ is derived (A11en, 1973), where $\mathrm{Ne}_{\mathrm{e}}$ is the electron number density and $\mathrm{V}$ is the volume of the envelope. Since He II 4686 emission line was very strong (Blair et a1.,1983), an electron temperature $20000 \mathrm{~K}$ is used. The observation of Viotti et a1. (1984) was made at phase 0.96 (June 27,1980 ), that is nearly on the phase of the maximum $\mathrm{U}$ luminosity. Lower values will be found in other phases. In the $U$ band the $f-f$ emission is about 2.6 times as bright as the cool component (A1len, 1973). Infrared photometries showed that the luminosity of the cool component is stable (Viotti et al.,1983), therefore the maximum amplitude of the light variation in the $U$ band is given as $2.5 \times \log ((2.6+1) / 1)$ $=1.4 \mathrm{mag}$., which is consistent with the observed results (Meinunger, 1979; 01iversen and Anderson, 1982). Further sources of the light variation, for example eclipsing phenomena, are not necessary. The variation in the $\mathrm{V}$ band should be less that $0.2 \mathrm{mag}$, which may correspond to the nearly 
stable V magnitude (Meinunger, 1979; Luthardt, 1985).

Since we assumed that the intensity of the $f-f$ emission varies according to a variation of mass transfer, the envelope should be included in the Roche lobe of the hot component: $\sim 130 R_{\odot}$. This radius is derived using the formula of Paczyński (1971) under the assumption that the masses of the primary and the secondary are $4 \mathrm{M}_{\odot}$ and $1 \mathrm{M}_{\odot}$, and the orbital period is 554 days. If the electron density in the envelope is $10^{10} \sim 10^{11} \mathrm{~cm}^{-3}$, which is suggested from the lack of the forbidden lines (Blair et al., 1983 ), this requested condition is satisfied. In the case of $\mathrm{N}_{e}=5 \times 10^{10}$ $\mathrm{cm}^{-3}$ we have a radius $60 \mathrm{R}_{\odot}$ and a mass $1 \times 10^{-8} \mathrm{M}_{\odot}$. The mass accretion rate onto the hot component is estimated to be $\sim 10^{-\top} \mathrm{M}_{\odot} /$ year (Iijima, 1986). The mass transfer rate in the binary system seems to be high enough to maintain the envelope.

\section{Conclusion}

The light variations of AG Dra may be due to a variation of $f-f$ emission from a gaseous envelope. The envelope, however, may not pulsate by itself, but probably varies according to a variation of mass transfer rate in the binary system. The emission measure of the envelope at a light maximum is about $6.5 \times 10^{59} \mathrm{~cm}^{-3}$ and a probable radius and a mass are $60 \mathrm{R}_{\odot}$ and $1 \times 1.0^{-8} \mathrm{M}_{\odot}$. The maximum amplitudes of the 1 ight variation in the $U$ and the $\mathrm{V}$ bands are 1.4 and 0.2 mags., which are consistent with the observed results (Meinunger, 1979; Luthardt, 1985).

I wish to thank Profs. L. Rosino, R. Barbon and Dr. F. Sabbadin for careful reading of the manuscript and for useful suggestions.

\section{References :}

Allen, C.W.: 1973, Astrophysical Quantities, The Athlone Press, London Anderson, C.M., et a1.: 1981, Astrophys. J. (Lett.) 24], L 127

Anderson, C.M., et a1.: 1982, in The Nature of Symbiotic Stars, IAU Coll. No. 70, eds. M. Friedjung, R. Viotti, D. Reide1 Pub1. Co., p. 117 Belyakina, T.S.: 1969, Izv. Krymskoj Astrofiz. Obs. 40, 39 (in Russian) Belyakina, T.S.: 1983, Izv. Krymskoj Astrofiz. Obs. $\overline{\overline{6}} \overline{\overline{\overline{8}}}, 108$ (in Russian) Blair, W.P., et a1.: 1983, Astrophys. J. Supp Z. 53 , $5 \overline{\overline{7}} \overline{3}$

Boyarchuk, A.A.: 1969, in Non-periodic Phenomena in Variable Stars, ed.

L. Detre, Academic Press, Budapest, p. 395

Doroshenko, V.T., Nikolov, N.S.: 1967, Soviet Astron. 11, 453

Iijima, T.: 1987, in Proceedings of IAU Col1. No. 93 on Cataclysmic

Variables, ed. H. Drechsel.

Kenyon, S.J., Webbink, R.F.: 1984, Astrophys. J. $2 \underline{\underline{7}} \underline{\underline{9}}, 252$

Luthardt, R.: 1985, Inf. Buzl. Variable Stars No. $=\overline{2} \overline{7} 89$

Meinunger, L.: 1979, Inf. Bull. Variable Stars No. 1611

Oliversen, N.A., Anderson, C.M.: 1982, in The Nature of Symbiotic Stars,

IAU Coll. No. 70, p. 177

Paczyński, B.: 1971, Ann. Rev. Astron. Astrophys. 9,183

Taranova, O.G., Yudin, B.F.: 1982, Soviet Astron. $\underline{\overline{2}} 6,57$

Viotti, R., et al.: 1983, Astron. Astrophys. $119, \overline{\overline{2}} \overline{\overline{8}} 5$

Viotti, R., et al.: 1984, Astrophys. J. $2 \frac{283}{=}, \overline{2} \overline{2} \overline{6}$ 